\title{
Development of a concept design process for an automotive body structure using a knowledge-based database
}

\author{
Won yong KI*, Seung-Jin HEO**, Dae-Oh KANG***, Hong Jae YIM**, Kyung won LEE****, \\ Jung ho KIM**** and Chang kun LEE**** \\ *Graduate School of Automotive Engineering, Kookmin University, 77 Jeongneung-ro Seonbuk-gu, Seoul, 136-702, Korea \\ **School of Automotive Engineering, Kookmin University, 861-1 Jeongneung-dong Seonbuk-gu, Seoul, 136-702, Korea \\ E-mail: sjheo@kookmin.ac.kr \\ ***Institute of Vehicle Engineering, 8 Hangang-daero 43-gil, Yongsan-gu, Seoul, 140-753, Korea \\ ${ }^{* \star * *}$ GM Korea, 199-1 Cheongcheon-Dong, Bupyeong-Gu, Incheon, 403-714, Korea
}

Received 1 February 2016

\begin{abstract}
The purpose of this study is to propose a concept design process for an automotive body structure using technical information on the major joints and members of vehicles. First, in order to collect the technical information on major joints and members, 43 vehicles were selected using benchmark data. The collected technical information for the selected vehicles was the cross sectional shapes of each joint and member which were used for the analysis of joint stiffness, crashworthiness and static stiffness of the member to make a database along with cross section properties. This study applied a 'What If Study' technique to perform a concept design of an automotive body using the analyzed information and selected cross section meeting the design objectives. The criteria for the selection of the cross section were defined by subdividing the defined design objectives of an automotive body structure and constraints into members and joints. In order to configure an analysis model of an automotive body structure using the selected cross section, a shape parametric model was used and static stiffness, dynamic stiffness and crashworthiness were assessed to evaluate the configured automotive body structure. The evaluation result showed that the crashworthiness and static/dynamic stiffness were improved by $10 \%$ and $5 \%$ respectively compared to an existing body structure. In addition, the weight of the body structure was reduced by $4.2 \%$. Through this study, the process that can rapidly and effectively derive and evaluate the concept design of an automotive body structure was defined. It is expected that, henceforth, this process will be helpful for the study of automotive body structures.
\end{abstract}

Key words : Body structure design, Knowledge based design, Conceptual design, What-if design, Cross-section database

\section{Introduction}

In general, an automotive body structure development process is divided into three phases: the development plan definition phase, the concept design phase, and the detail design phase. However, as the requirements for automotive body structures are constantly increased and diversified, strategies for the development of an automotive body structure continue to change. (Donndelinger, J, 2006) Figure. 1 clearly shows such tendencies. It can be seen that most automobile companies have established a strategy that will see model development increase by an average of $67 \%$ over the next four years, a marked increase over the past four years. As this strategy suggests, even strategies for the design of automotive body structures is changing, most notably by improving the design accuracy at the concept design phase. (Berylls strategy 
advisors, 2012)

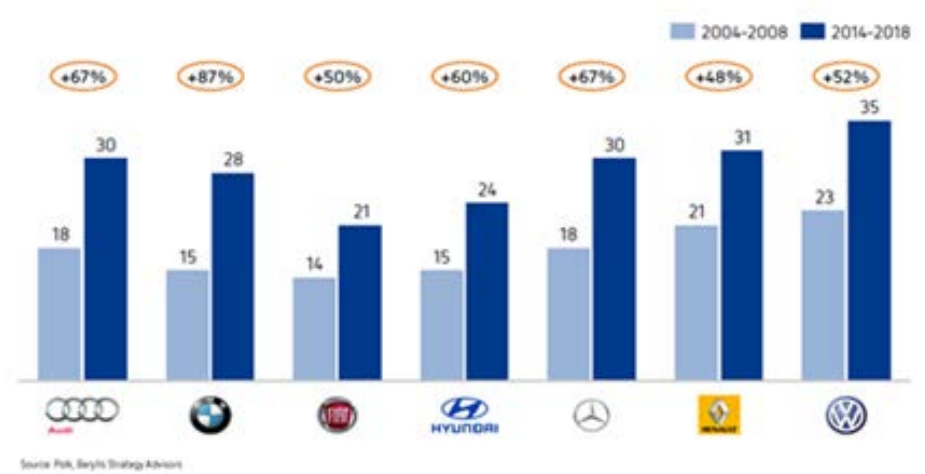

Fig. 1 Sum of new model launches for selected car brands

As can be seen in figure 2, the design freedom at the concept design phase is higher than in other design phases. Therefore, various design ideas can be applied at the concept design phase and a design meeting various requirements can be derived by examining the performance of each application plan. If the design accuracy increases at the concept design phase, the number of design changes can be reduced at the detail design phase, leading to reduced time of design development and cost saving. This study describes the difference between a new design methodology and the existing design methodology by investigating the design methodology to improve accuracy at the proposed concept design phase and by proposing a new design methodology. (David G. Ullman, 2003) In Fig.2, BIW means body in white, which refers to the stage in automotive design or automobile manufacturing in which a car body's sheet metal components have been welded together. BIW is termed before painting \& before moving parts (doors, hoods, and deck lids as well as fenders), the motor, chassis sub-assemblies, or trim (glass, seats, upholstery, electronics, etc.) have been assembled in the frame structure.

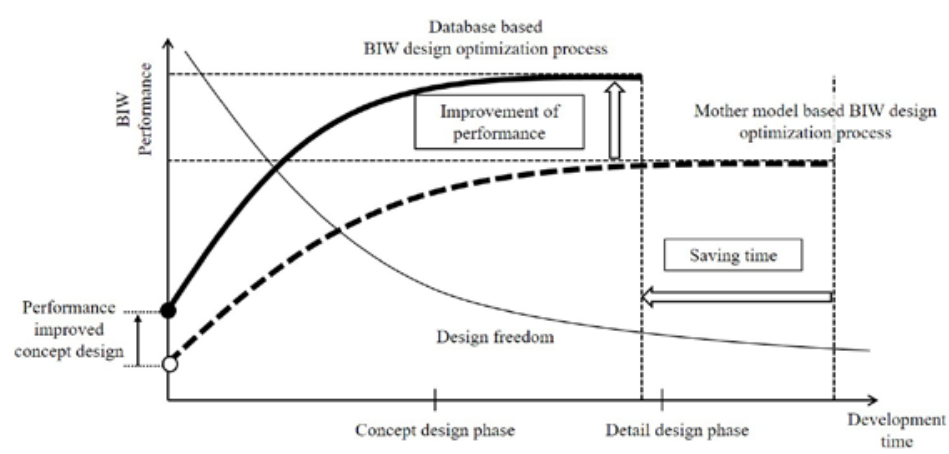

Fig. 2 Status of product knowledge level, degree of freedom for decision and cost responsibility at vehicle development process

\subsection{Investigation of documents related to the concept design process of an automotive body structure}

In the prevalent concept design theories, a high fidelity concept model is constructed using three design methods: methods based on the predecessor model, methods "from scratch". (Donders, Stijn et al., 2009) Methods based on predecessor models could achieve higher design efficiency compared with other methods, since predecessor models include essential design factors such as load path information of the load case. Mitsubishi Motors evaluated the structural performance of a vehicle model in terms of the joint stiffness. Each joint is decomposed in the finite element model and assigned a separate set of material properties. Evaluation of the car body structure based on the optimum joint stiffness showed a superior performance relative to the baseline model without a weight penalty. (Martez Kiani et al., 2014) Marco Danti designed a new concept model using morph volume methodology. The new concept model is constructed by modification of the predecessor model's section shape and structure length. To evaluate the concept model, static stiffness and dynamic stiffness are selected as the load case. (Danti, Marco, et al., 2010) Duddeck used the SFE CONCEPT, which is an engineering tool using implicit parameterization technique, for his concept model construction. The parameterized 
model was based on the reference model, and the design target was crashworthiness improvement. (Duddeck, F. and H. Zimmer, 2012) Long reports a study of predecessor joints, linking geometrical characteristics to the stiffness performance. For a vehicle stiffness target, the most suitable joint can then be selected. (L, Long, 1998) The "from scratch" method is used at the very beginning of the design process. It could be classified as topology optimization and functional layout design of load-carrying components. Topology design optimization is shape and size optimization by eliminating material from the admissible design space. WorldAutoSteel, the automotive group of the World Steel Association, used topology optimization for skeleton structure design in an FSV program. The FSV (Future Steel Vehicle) program developed this initial structure by considering three longitudinal load cases, two lateral load cases and one vertical load case, as well as investigations about bending and torsional static stiffness. Though the topology optimization was able to provide an initial starting point for the FSV's geometry, it is limited by its static approximation of dynamic crash loads and does not consider grade variations of the sheet metal within the structure. (World Auto steel, 2011) Also, the functional layout design used a simple geometric description of functional elements such as beam, joint and panel. Toyota proposed first order analysis to determine basic layouts based on structural mechanics. It included a topology optimization method using function oriented elements and a joint analysis method considered nonlinear behavior using a simplified model. (Nishigaki et al., 2001) S. Dondersa suggested a reduced beam and joint modeling approach to analyze and optimize the global bending and torsion modes of a vehicle body. (Donders, Stijn et al., 2009) BMW proposed a concept modeling process which is based on the FE "Beams and Shells" concept model, with arbitrary beam cross sections, which is used for early concept phase car body structural optimization with a special focus on the NVH (Noise, Vibration, Harshness) performance. (A. Moroncini et al., 2012)

\subsection{Methodology}

The design methodology proposed by this study involves deriving a design that's close as possible to the design objectives by examining various design problems at the initial design phase (concept design phase) with high design freedom. Since major joints and members have an effect on automotive body structure performance, this study propose a concept design process using technical information on the major joints and members of vehicles. By joints and members selection considered automotive body structure requirements, automotive body structure satisfied design requirements would be achieved in at the initial design phase. Therefore, this study suggest innovative concept design process using knowledge based database, which included design know-how and performance evaluation result. Suggested design process consisted of two phases as shown Figure 3: the first phase is construction of knowledge based database and the second phase is concept design phase.

In construction of knowledge based database phase, in order to collect the technical information on major joints and members, 43 vehicles were selected using benchmark data. The collected technical information for the selected vehicles was the cross sectional shapes of each joint and member which were used for the analysis of joint stiffness, crashworthiness and static stiffness of the member to make a database along with cross section properties.

In concept design phase, this study applied a 'What If Study' technique to perform a concept design of an automotive body using the analyzed information and selected cross section meeting the design objectives. The criteria for the selection of the cross section were defined by subdividing the defined design objectives of an automotive body structure and constraints into members and joints. Since design objectives were defined as automotive body structure mass reduction considered automotive body structure requirements (crashworthiness, static stiffness, dynamic stiffness), the cross sections, which are lighter weight and better joint and member performance than reference model, are selected.

In order to configure an analysis model of an automotive body structure using the selected cross section, a shape parametric model was used and static stiffness, dynamic stiffness and crashworthiness were assessed to evaluate the configured automotive body structure.

This study describes the method to establish a database for each phase in Chapter 2, the method to carry out the design using the database in Chapter 3, and the conclusions of this study in Chapter 4. 
Fig. 3 Design process using a knowledge-based database

\section{How to establish the database}

\subsection{Establishment of a database for automotive body structures}

This study used the benchmark data of automotive body structures in order to establish a database which was the core of the proposed design methodology. The information on a total of 43 vehicles was used for database. This study collected a variety of information on the year of manufacture of the vehicles from 2008 to 2014 in order to allow analysis of annual design changes of the same types of vehicles, and built a database of the information on the performance of an automotive body structure through the crashworthiness evaluation index (full frontal crash and side crash) of each vehicle.

\subsection{Establishment of a database for key joints and members}

In general, the subsystem is divided into bisections vertically (upper / under) or trisections (front / middle / rear) horizontally. However, this study defined the subsystem as the key joints and members (A pillar upper joint, A pillar middle joint, A pillar lower joint, B pillar upper joint, B pillar lower joint, C pillar upper joint, front member, and rear member) as shown in figure 4. In addition, the performance index related to the performance of an automotive body structure was defined in order to analyze the performance of each joint and member. This study evaluated the axial compression characteristics and bending collapse characteristics related to the crashworthiness of a vehicle, as well as the static stiffness (bending stiffness, torsional stiffness) and joint stiffness, which were related to the body structure stiffness, and made a database of these values. In addition, the manufacturing cost was estimated when applying each material as well as its performance in order to analyze the difference in the performance of mild steel and advanced high strength steel. 


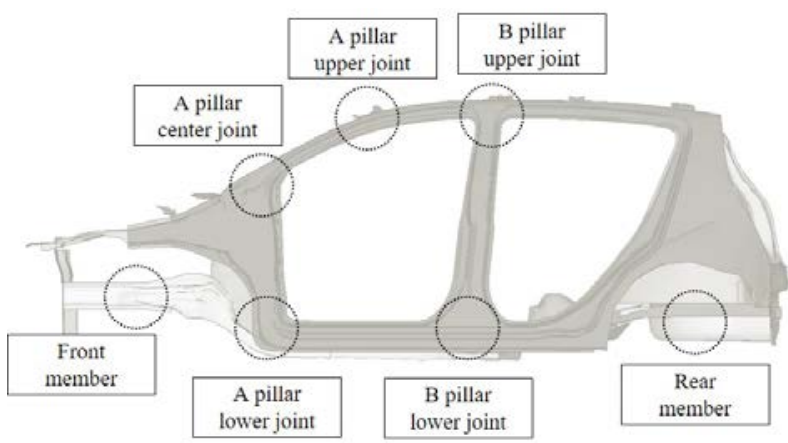

Fig.4. Selected body joint part and member for cross section shape database generation

\subsubsection{Axial compression characteristics}

In this study, buckling load (peak load) is used for axial compression characteristics of members. For more detail analysis, as shown figure 5, eigenvalue analysis was performed to predict the point at which buckling occurs in order to analyze the structural behavior due to structural instability. By performing post buckling analysis applying only up to the tertiary mode as the resultant value of the mode shape for which eigenvalue analysis was completed, it was possible to predict the geometric characteristic and position at which buckling occurs.
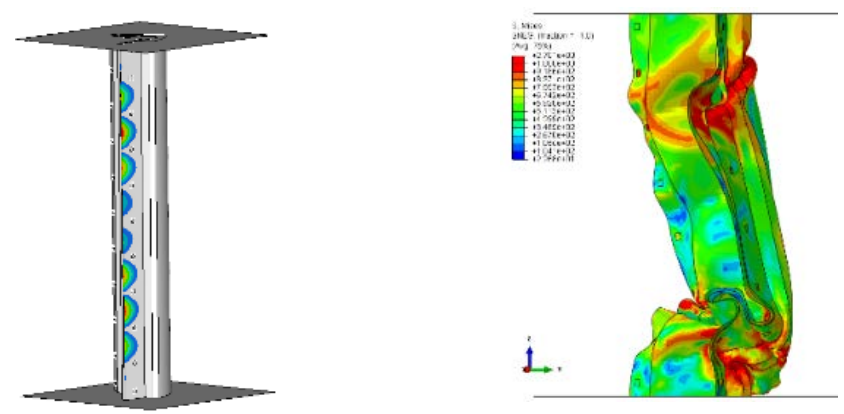

Fig.5. Example of buckling analysis

\subsubsection{Bending collapse characteristics}

This study analyzed the bending collapse characteristics of the key members in order to evaluate the bending stress of the key members which occurs due to a collision of the automotive body structure. The Kecman's analysis has mainly been used for the analysis of the bending collapse characteristics. Kecman performed experiments with cantilever beam bending by completely fixing one end of a beam. Since the bending collapse equation derived by him has coefficients that must be determined through experiments, it can be called a semi-empirical formula. Therefore, this study made an experimental device the same as the one used for the experiment proposed by Kecman as shown in figure 6 to evaluate the bending collapse characteristics of each member. (Kecman, 1979)

- Actuator velocity : $0.3 \mathrm{~mm} / \mathrm{s}$ (Quasi-static)

- Displacement : $120 \mathrm{~mm}$, time $=400 \mathrm{~s}$

- Test piece : Square

$(125 \mathrm{~mm} * 70 \mathrm{~mm} * 1000 \mathrm{~mm}, 2 \mathrm{t})$

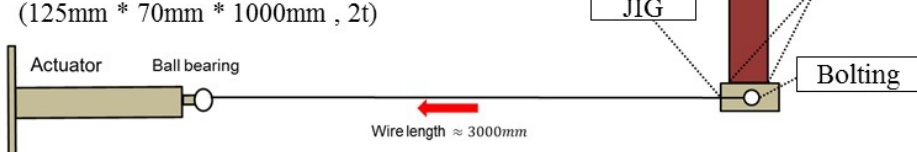

Fig.6. Bending collapse test setting

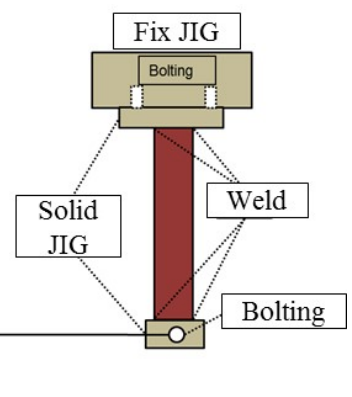

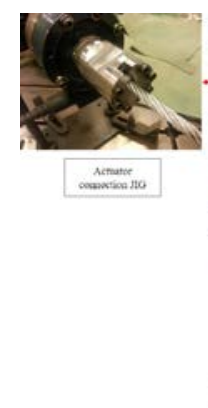

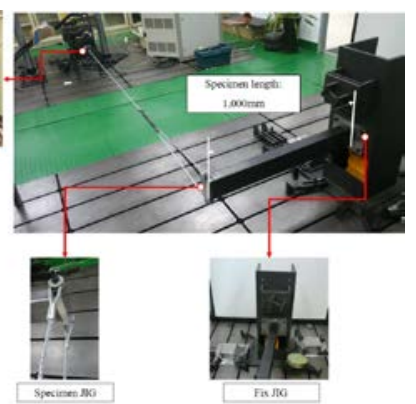




\subsubsection{Static stiffness}

It is important to define the boundary condition in order to evaluate the static stiffness of each member since a simple evaluation of the static stiffness of a cantilever with one end being completely fixed may derive a result different to the actual behavior of an automotive body structure. Therefore, this study analyzed the bending characteristics by establishing an analysis model in the shape of a beam with both ends being simply supported. Also, in the case of the torsional stiffness, the static torsional characteristics were analyzed by defining the boundary condition with the same condition. (Malen, Donald E., 2011)

\subsubsection{Joint stiffness}

The rotational stiffness of a joint has a significant influence on the natural frequency of a vehicle due to local deformation. Therefore, various studies have been performed concerning the joint stiffness, and as a result, various methods to predict joint stiffness using a formula as well as the methods to calculate joint stiffness taking nonlinearity into consideration are presented. However, this study built key joint models and derived the joint stiffness through linear static analysis and nonlinear quasi-static analysis using the tip moment method. The analysis conditions were derived as shown in figure 7 by extracting joints from the vehicle model and constraining the six degrees of freedom at one end of a joint branch.

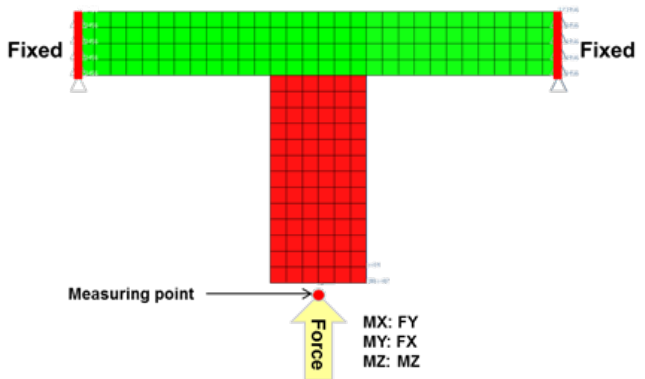

Fig. 7. Boundary condition for joint stiffness analysis

In addition, the load was defined according to the length of each joint branch and the defined load and moment were applied in the direction of the set local coordinate respectively, and then the rotation angle of each direction was measured at the point to which the load is applied. The measurement result was substituted to Eq. (1) to obtain the rotational stiffness in each direction.

$$
K_{x}=\frac{M_{x}}{\theta_{x}}, K_{y}=\frac{M_{y}}{\theta_{y}}, K_{z}=\frac{M_{z}}{\theta_{z}}
$$

\subsubsection{Manufacturing costs}

Steel manufacturers and automobile manufacturers now perform various analyses of production cost and vehicle weight, providing countermeasures against these problems. Therefore, this study calculated and indexed material cost, machining cost and production cost using the materials actually applied to automotive body structures. Table 1 shows the manufacturing process and evaluation items for the unit cost of production. For the unit price of each material and manufacturing process cost for manufacturing cost calculation, this study referred to the result of analysis performed by WorldAutoSteel.

Table 1. Manufacturing processes and operations sequence

\begin{tabular}{c|c|c}
\hline \hline & Stamping & Tailored blanking stamping \\
\hline Material price & Steel material price (Mild steel, AHSS) & Steel material price (Mild steel, AHSS) \\
\hline Operation \#1 & Blanking & Blanking \\
\hline Operation \#2 & Stamping & Welding \\
\hline Operation \#3 & & Stamping \\
\hline
\end{tabular}


Cost evaluation includes the factors of Energy Cost, Equipment Cost, Tooling Cost and Material Cost, which are shown in figure 8.

\begin{tabular}{|l||l|l|}
\hline Energy & Equipment & Tooling \\
$\checkmark$ Ordinary stamping & $\checkmark$ Ordinary stamping & $\checkmark$ Stamping \\
- Blanking + Stamping & - Blanking + Stamping & - Cost : $\$ 33.6 /$ unit \\
- Cost $: \$ 0.05 /$ unit & - Cost $: \$ 27.92 /$ unit & Material \\
$\checkmark$ Tailored blanking stamping & $\checkmark$ Tailored blanking stamping & $\checkmark$ Mild steel $: \$ 60 /$ ton \\
- Blanking + Welding + Stamping & - Blanking + Welding + Stamping & $\checkmark$ AHSS : $\$ 70 /$ ton \\
- Cost $: \$ 0.35 /$ unit & - Cost $: \$ 209.66 /$ unit & \\
\hline
\end{tabular}

Fig.8. Detailed assumptions for manufacturing cost analysis

"Energy Cost" includes the cost of electricity needed for manufacturing, "Equipment Cost" refers to the cost needed to establish the manufacturing process system, "Tooling Cost" refers to the machining cost, and "Material Cost" refers to the cost of mild steel and advanced high strength steel (AHSS). The production cost was calculated as production investment taking into consideration the general stamping technique and the tailored blanking stamping technique. In the case of the stamping technique, the machining cost was estimated by dividing the stamping technique into Blanking, Welding and Stamping and considering the cost for the power consumption by each process as well as the duty cycle of the press. Each production cost was estimated by assuming that 250,000 products would be produced. (American iron \& Steel institute,1998)

\subsection{Establishment of the cross section database}

The cross-sectional shapes of the beam elements are often used in automotive structures. Such cross-sections are manufactured by welding together a number of pressed sheet metal elements. The cross-sectional area $S$, moments of inertia Iy and Iz, area product of inertia Iyz, principle inertia directions $\Phi$ influence the stiffness of the structure. These criteria are calculated using the following formula. (Izui, K. et al., 2003)

$\mathrm{S}=\int d A$

$\mathrm{I}_{y}=\int z^{2} d A$

$\mathrm{I}_{z}=\int y^{2} d A$

$\mathrm{I}_{y z}=\int y z d A$

$\phi=\frac{1}{2} \tan ^{-1}\left(\frac{-2 I_{y z}}{I_{z}-I_{y}}\right)$

The thin wall closed section torsional constant with uniformed thickness $\mathrm{J}$ is given by:

$\mathrm{J}=\frac{4 A^{2}}{\sum_{i} \frac{S_{i}}{t_{i}}}$

where $\mathrm{A}$ is the area enclosed by the section, $\mathrm{S}$ is the section perimeter, and $\mathrm{t}$ is thickness (Malen, Donald E., 2011). Also, the plastic section modulus is selected for plastic behavior evaluation. The plastic section modulus is the sum of the areas of the cross section on each side of the plastic neutral axis multiplied by the distance from the local centroids of the two areas to the plastic neutral axis. This factor is also used for shape factor calculation with elastic section modulus. 


\section{Automotive body structure concept design using the database}

\subsection{Design Problem Formulation}

The virtual design (What-if Study) used to derive the optimum combination of design parameters can be expressed in the minimized form of the optimum equivalent functional when there is conflict between the design parameters in the combination of design parameters that minimize each performance index. Therefore, as a result of the What-If-Study, the combination that configures the minimum value in the effect analysis becomes the 'optimum combination' regardless of the minimum problem and maximum problem. (Institute of Design Optimization, 2011)

The mathematical equivalent functional as shown in Eq. 8 is used to derive the optimum combination of the design parameters. The $f(x)$ is objective function, which is minimized mass of body structure in this study. And $x$ is design variables which are cross section of each joint and member in this study. And $\mathrm{g}(\mathrm{x})$ are constraints, which are 5 joint and member performance. So, the cross sections, which are better performance and lighter than initial design, are selected in database.

$\operatorname{MIN~} \mathrm{f}(x)$

Where

$$
g_{i}(x)>g_{i}^{o}, i=1,2,3,4,5
$$

\subsection{Result of Concept Design and Establishment of Analysis Evaluation Model}

Table 2. shows the result of the concept design. 4 cross sections are selected by what if study. In case of these cross sections, other cross sections which satisfied design constraints are included in database. But in case of other 6 cross sections, even though there are other cross sections which are satisfied with constraints, reference model is selected considering mass.

Table 2. Result of concept design by what if study

\begin{tabular}{ccl}
\hline & & Result of concept design \\
\hline $\mathbf{1}$ & Front roof cross member cross section & 2012 F-B model \\
\hline $\mathbf{2}$ & Roof rail cross section & 2012 M-B model \\
\hline $\mathbf{3}$ & A-pillar upper cross section & 2013 M-A model \\
\hline $\mathbf{4}$ & A-pillar middle cross section & $\begin{array}{l}* \text { Even though there are other } \\
\text { cross sections which are } \\
\text { satisfied with constraints, } \\
\text { reference model is selected } \\
\text { considering mass. }\end{array}$ \\
\hline $\mathbf{6}$ & A-pillar lower cross section \\
\hline $\mathbf{7}$ & Rocker panel cross section & \\
\hline $\mathbf{9}$ & B-pillar upper cross section & 2013 R-C model \\
\hline $\mathbf{1 0}$ & B-pillar lower cross section &
\end{tabular}

From the results of the performance comparison (as shown figure 9), it was found that there is a difference in the cross sectional characteristics compared to the reference cross section and that the performance of the member and joint also shows a tendency similar to the cross sectional characteristics. An analysis model was established to evaluate the performance for the body structure concept design by applying the selected cross section to the body structure. In order to establish the analysis model, a parametric model was established using the SFE CONCEPT, which is an engineering tool using implicit parameterization technique. The parametric model signifies a model with the design parameter and model shape directly connected. Figure 10 shows the established model. Selected cross sections are applied to established parametric model, and then joints and members are changed. 


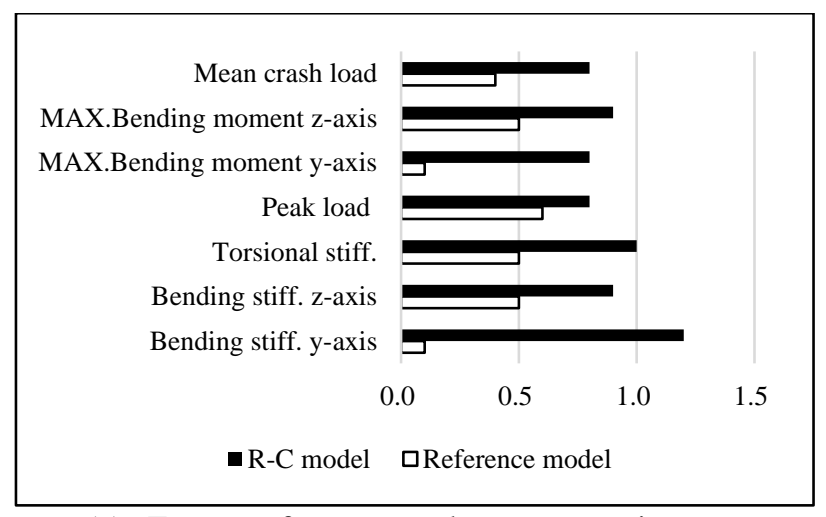

(a) Front roof cross member cross section

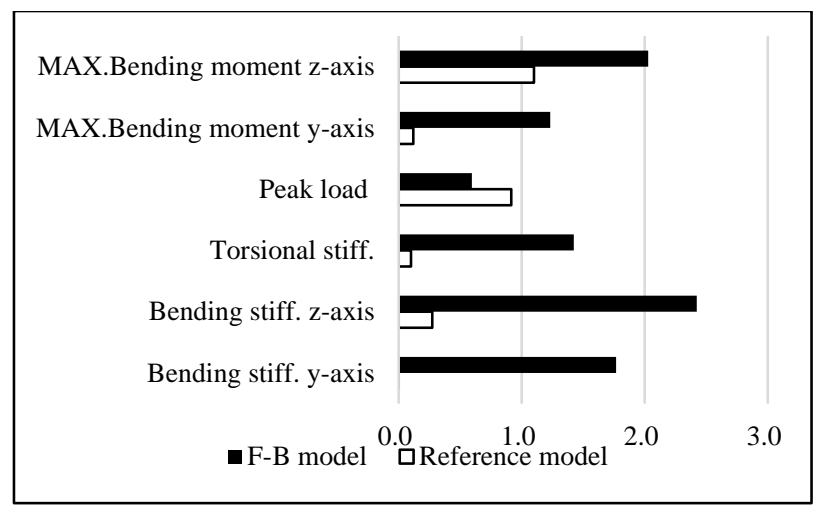

(c) A-pillar upper cross section

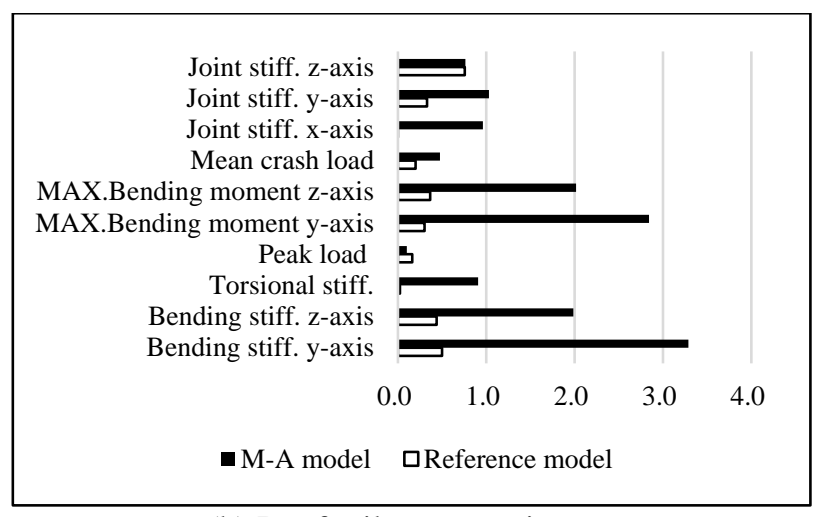

(b) Roof rail cross section

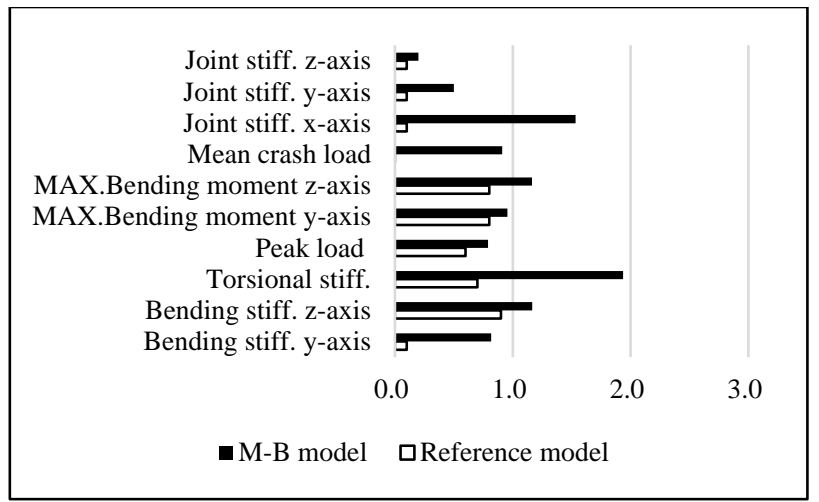

(d) Front member cross section

Fig. 9. Performance of the member and joint to which each selected cross section

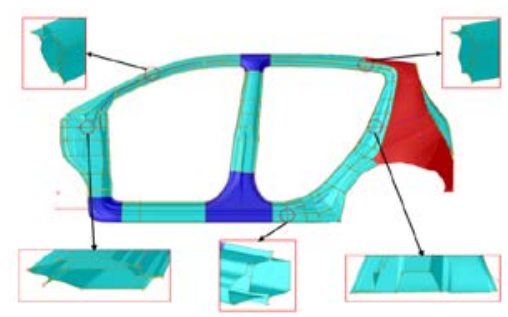

(a) Linked cross sections to parametric model

Fig. 10. Constructed automotive body structure parametric model using SFE CONCEPT and linked cross sections description

\subsection{Automotive Body Structure Concept Design Evaluation Result}

For the evaluation of the body structure, this study evaluated static stiffness and dynamic stiffness and checked the performance changes compared to the reference body structure. Table 3 shows the evaluation result.

Table 3. Performance evaluation result

\begin{tabular}{c|c|c}
\hline \hline & Performance index & Concept model \\
\hline \multirow{3}{*}{ Static stiff. } & Bending stiff. & $7 \%$ improved \\
\cline { 2 - 3 } & Torsional stiff. & $3 \%$ improved \\
\cline { 2 - 3 } & $1^{\text {st }}$ Torsional mode & $2 \%$ improved \\
\hline \multirow{3}{*}{ Crashworthiness } & Full frontal & $14 \%$ improved \\
\cline { 2 - 3 } & Side crash & $15 \%$ improved \\
\cline { 2 - 3 } & Roof crush & $12 \%$ improved \\
\hline & Cost & $2 \%$ increased \\
\hline
\end{tabular}


It was found from the evaluation result that bending stiffness and torsional stiffness and dynamic stiffness increased by $7 \%$ and $3 \%$, respectively. In the case of crashworthiness, the full frontal crash, side crash and roof crush increased by $14 \%, 15 \%$ and $12 \%$, respectively. And the cost is increased by $3 \%$. The small area cross section, compared with reference model, were selected. Therefore the incensement of cost was small.

And the reason for the significant increase in crashworthiness compared to static/dynamic stiffness is that the selected cross sections include the cross section to which advanced high strength steel (AHSS) was applied. Figure 11 shows the properties applied to the concept design.

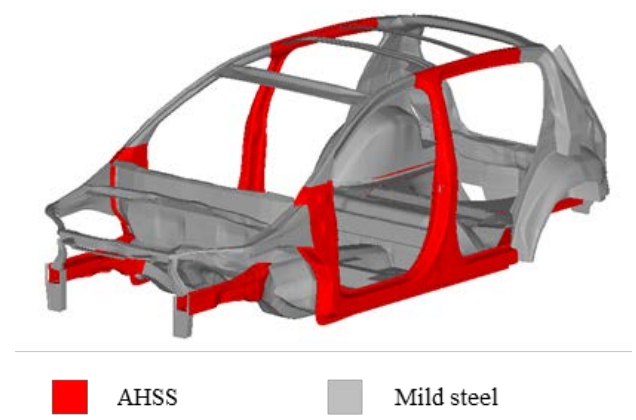

Fig. 11. Material distribution of concept model

In addition, Table 4 shows the results of the comparison of the analysis time and degree of weight reduction between the concept design method through the existing multi-disciplinary constrained optimum design and the methodology proposed by this study. The comparison results show that the weight reduction effect increased and the design time was reduced by $50 \%$.

Table 4. Performance evaluation result

\begin{tabular}{c|c|c}
\hline \hline & MDO & Conceptual Design Knowledge based Cross-section Database \\
\hline Mass Reduction Rate [\%] & $-1.4 \%$ & $-4.2 \%$ \\
\hline Violated Constraints & 0 & 0 \\
\hline Solving Time (week) (32 CPU) & 2 & 1 \\
\hline
\end{tabular}

\section{Conclusion}

This study proposed a concept design methodology using a database for the structural performance and shape of the key joints and members configuring an automotive body structure in order to improve design accuracy at the concept design phase. This study used the information on cross sections of 43 mass produced vehicles and calculated the characteristics of each cross section to make a database. In addition, this study defined the key joints and members as a subsystem of an automotive body structure, and analyzed the crashworthiness and static stiffness of each joint and member to make a database, and made it possible to predict the performance of the subsystem for each cross section when performing concept design by establishing the relationship with the established cross sectional characteristics. Lastly, it was made possible to predict the performance of an automotive body structure for cross sections by establishing the relationship between the subsystem and body structure. In order to perform the concept design of an automotive body structure using the database established as such, the design problems that defined the purpose of body structure design and constraints were formulated, and a What-If study technique was applied to select a cross section, which is a design parameter, according to the defined design problems. A cross section meeting the design purpose was then selected and in order to configure an automotive body structure with the selected cross section, a shape parameter model was used. In order to evaluate the performance of the configured body structure, the crashworthiness and static/dynamic stiffness performance were evaluated and by comparing the results with the reference automotive body structure, the reliability of the body structure established by the design methodology proposed by this study was secured. 


\section{Acknowledgement}

This research was supported by GM Korea and the MSIP(Ministry of Science, ICT and Future Planning), Korea, under the C-ITRC(Convergence Information Technology Research Center) (IITP-2016-H8601-16-1005) supervised by the IITP(Institute for Information \& communications Technology Promotion)

\section{References}

American iron \& Steel institute (1998), Ultra Light Steel Auto Body final report.

Berylls strategy advisors (online), available from http://www.berylls.com (accessed 2012).

Danti, M., Meneguzzo, M., Saponaro, R., \& Kowarska, I., Multi-objective optimization in vehicle concept modeling, In Proceedings of the International Conference on Noise and Vibration Engineering ISMA (2010), pp. 4095-4108.

Donndelinger, J. A decision-based perspective on the vehicle development process. Decision Making in Engineering Design (2006), pp.217-226.

Donders, S., Takahashi, Y., Hadjit, R., Van Langenhove, T., Brughmans, M., Van Genechten, B., \& Desmet, W., A reduced beam and joint concept modeling approach to optimize global vehicle body dynamics. Finite Elements in Analysis and Design, Vol.45, No.6 (2009), pp.439-455.

Duddeck, F., \& Zimmer, H., New achievements on implicit parameterization techniques for combined shape and topology optimization for crashworthiness based on SFE CONCEPT. Shape and Technology Optimization for Crashworthiness, ICRASH2012. (2012)

Institute of Design Optimization, Inc. Version 2.0 User’s Manual for EasyDesign (2011)

Izui, K., Nishiwaki, S., \& Yoshimura, M., An Optimal Cross-Sectional Design Method for Automotive Body Frames). SAE Technical Paper, (2003), Paper No. 2003-01-2782

Kiani, M., Motoyama, K., Rais-Rohani, M., \& Shiozaki, H., Joint stiffness analysis and optimization as a mechanism for improving the structural design and performance of a vehicle. Proceedings of the Institution of Mechanical Engineers, Part D: Journal of Automobile Engineering, (2014). Paper No. 0954407013516107

Kecman, Dušan. Bending collapse of rectangular section tubes in relation to the bus roll over problem, (1979)

Long, Luohui , Design-oriented translators for automotive joints. Diss. Virginia Polytechnic Institute and State University, (1998)

Malen, Donald E., Fundamentals of automobile body structure design. Vol. 394, (2011), SAE Technical Paper

Moroncini, A., Cremers, L., \& Baldanzini, N.,Car body concept modeling for NVH optimization in the early design phase at BMW: a critical review and new advanced solutions. In Proceedings of the International Conference on Noise and Vibration Engineering, (2012), ISMA 3809-3823

Nishigaki, H., Nishiwaki, S., Amago, T., Kojima, Y., \& Kikuchi, N.,First order analysis-new CAE tools for automotive body designers, SAE Technical Paper, (2001), Paper No. 2001-01-0768

Pan, Z., Wang, X., Teng, R., \& Cao, X., Computer-aided design-while-engineering technology in top-down modeling of mechanical product, Computers in Industry, No.75, (2016), pp. 151-161.

Ullman, G. D., The Mechanical Design Process third edition (2003), McGraw-Hill.

World Auto steel (2011) Future Steel Vehicle final report 